\title{
423 - Visual Hallucinations in Parkinson Disease and Dementia with Lewy Bodies: a review
} Joana Regala ${ }^{1}$, Camila Nóbrega $^{2}$, João Reis ${ }^{3}$

1- Centro Hospitalar Psiquiátrico de Lisboa (CHPL), Lisbon, Portugal.

2- Department of Neurology, Centro Hospitalar Psiquiátrico de Lisboa (CHPL), Lisbon, Portugal.

3- Department of Geriatric Psychiatry, Centro Hospitalar Psiquiátrico de Lisboa (CHPL), Lisbon, Portugal.

Visual Hallucinations (VH) are a common psychiatric symptom of Parkinson's Disease (PD) and Dementia with Lewy Bodies (DLB). According to the Perception-Attention Deficits Model, VH stem from impaired perceptual (bottom-up), including dorsal (DVS) and ventral visual streams (VVS), and attentional (topdown) processes, with a persistent Default Mode Network (DMN) activity and a dysfunctional imbalance of Dorsal (DAN) and Ventral Attentional Networks (VAN).

We review the literature on the neurobiological underpinnings of VH in DLB and PD, concerning overlapping and different mechanisms.

In DLB hallucinators, PET-scan findings of disconnection between higher and primary visual areas and dysfunctional recruitment of the VVS, corroborate other imaging and neuropathologic studies which document inferior longitudinal fasciculus damage and increased temporal lobe Lewy body (LB) pathology. The alteration of network topography is more pronounced in the DAN and DMN. The involvement of anterior cortical regions, clustering around attentional networks, is demonstrated in neuropathologic, volumetric and perfusion studies. VH severity closely correlates with anterior cingulate cortex and inferior temporal cortex hypoperfusion, structural changes in the DVS (superior parietal gyrus and precuneus) and increased diffusivity of the right thalamic projections to parieto-occipital cortices. Thalamocortical dysfunction alongside decreased cholinergic activity in reticular nucleus, which receive projections from the nucleus basalis of Meynert, seems to play a crucial role.

Both DLB and demented PD hallucinators have more frontal cortical atrophy, yet greater in DLB, supporting top-down mechanisms.

Regarding PD hallucinators, findings of cortical atrophy in inferior parietal lobule, cuneus, lingual lobule, and precentral gyrus correlated with hallucination scores. However, some studies did not evidence volumetric cortical differences between hallucinators and non-hallucinators. Moreover, LB are not necessarily present in hallucinators, suggesting other neuropathologic mechanisms in the genesis of $\mathrm{VH}$, namely altered neurochemical circuitry. Volume loss in pedunculopontine nucleus and right-thalamus support the hypothesis of a dysfunctional cholinergic brainstem control of the cortex. PET-scan studies discovered higher 5-HT2A receptor levels in the inferolateral temporal and prefrontal cortices. A greater nigrostriatal impairment is documented in the right-caudate of hallucinators. Therefore, $\mathrm{VH}$ may arise from an inability to activate DAN (in which caudate is involved) and consequent faulty visuo-perceptual processing by DMN and VAN. 